\title{
A language for qualia: on verbal interface of the phenomenal
}

\author{
Sergei Kuzeev \\ Doctoral School of Linguistics, Eötvös Loránd University, Budapest, Hungary
}

\begin{abstract}
Despite several decades of intense scrutiny, the problem of the "explanatory gap" remains one of the most topical issues in today's cognitive sciences. This paper argues that, if re-articulated as the (relative) ineffability of phenomenal properties of sensory experiences, it can become an object of linguistic treatment to a sensible effect. The paper proceeds from discussing the problem of ineffability at large to a brief analysis of the current accounts of phenomenal mental states. It then proposes a tentative descriptive framework for phenomenal judgments, i.e. statements involving reference to the speaker's qualia. The main argument of the paper consists in relativization of the ineffability thesis and in establishing that phenomenal contents can be communicated verbally via a special type of discursive units-phenomemes--by way of referencing relational properties of the sensory experiences in question. In the concluding section, the paper suggests that phenomemes constitute a narrative dimension and highlights the potential of further research on the subject for the pragmatics of communication, cognitive stylistics, and other areas of the language-related scholarship.
\end{abstract}

\section{Key words}

ineffability, phenomenal concepts, phenomenal judgments, phenomemes

\section{Introduction}

Since the time of ancient thinkers, there has been a lingering conviction that our public language is essentially incapable of conveying the entire intricacy of human inner lives. This is the kind of overstatement which we have to hold with nonetheless, as every now and then, in our everyday conversations as well as on the pages of distinguished literary works, do we encounter such signs of verbal failure. For those of the positivistic mindset, there is nothing mysterious about this unsayability, since they treat it as a lack of linguistic competence or as a deficiency in one's individual vocabulary. Others do not discard the problem so easily and assume that it owes to a certain incongruence between how the sensual domain and language work. But what rarely receives systematic attention is the actual linguistic interface of the said gap, that is, the mechanisms of overcoming the ineffability of those sensory contents that we typically find hard to verbalize.

In this essay, I make an attempt at re-articulating the problem of verbal closure as the relative ineffability of phenomenal mental states, i.e. of the non-propositional knowledge that we acquire when undergoing sensory experiences ("qualia"). I start with a general outline of the problem at hand and proceed to a brief discussion of subjectivity of mental states and its language-related effects. I then suggest that phenomenal states are only ineffable in the weak sense-insofar as their experiential qualities have to be addressed directly. Based on evidence from literary discourse (which is the principle realm of phenomenal contents organized as coherent text), I claim that we typically verbalize our phenomenology via relational descriptions which work, along the broad lines, similarly to (novel) metaphors and metonymies. I dub those analogy-based discursive units that refer to speaker's qualia as phenomemes, which can be categorized, primarily, along the axes of complexity, i.e. degree of reliance on available public concepts, and stability, i.e. reducibility to existing conceptual models.

The essay concludes with a brief examination of the use of phenomemes as a narrative technique that facilitates a more intimate engagement of readers with fictional texts and puts forth "mind reading" as a source of interpretative insights. This research is motivated by the relative scarcity of attention to the relations between language and qualia (with the notable exception of, for instance: Berta 2011; Carruthers 1996; Zlatev 2008), despite the manifest linguistic dimension of this problematic. The paper, thus, aims at devising a tentative working model for discussing ineffability of phenomenal mental states in the context of cognitive stylistics and discourse analysis.

\section{Ineffability of Mental States}

Wittgenstein's "Tractatus" ends, famously, in "whereof one cannot speak, thereof one must be silent" (2009: 82 ), and, as is the case with much of the Austrian philosopher's writing, there are many conflicting interpretations of the maxim - from extreme positivist to overtly mystical. However, according to the later exegesis of his oeuvre (Nordmann 2005: 144-154), here Wittgenstein asserts that there are truths about the world that, for one reason or another, our public language cannot conclusively convey. Simply said, and putting 
the metaphysical implications of this premise aside, it recognizes the boundaries of language with respect to at least some of our life experiences. When I think of, for instance, being immersed into the ethereal beauty of a musical etude, or of marveling at the grandeur of a natural landscape, or of simply enjoying a glass of wine on a quiet homely evening, I apperceive that verbalizing the feelings which these experiences entail is strangely problematic, and that I have to rely on those rough approximations of our common vocabulary--"amazement", "delight", "tranquility", "happiness", etc.—which hardly do justice to those mental contents, of which I am conscious through introspection. This engenders the intuition that our affective states, at least to a degree, are beyond the linguistic horizon: each time we try to communicate them, we are beset with their ineffability, fragility against the machinery of language.

This is the kind of complication that made Cordelia, King Lear's daughter from Shakespeare's play, lament: "Unhappy that I am, I cannot heave my heart into my mouth" (2009: 7), when asked to describe her love for the father. The problem that she was facing is not, strictly speaking, the lack of words, but the sublime realization that whatever we are able to say on account of our feelings is, at best, only a postiche of the complex impressionistic painting drawn by our senses in rich, elaborate strokes. This not only hints at a certain tension between the verbal and the non-verbal, but raises an uneasy dilemma: is language an adequate medium for communicating our sensory experiences? The trouble is, if we answer this question in the negative, we will find ourselves in another blind alley, for language remains the only medium that enables us to communicate our mental contents to a sensible effect: dancing, gesturing, or painting them may be spectacular activities which, however, can hardly be used as reliable and consistent tools of information transfer ${ }^{1}$. All (or most of) our thoughts and apprehensions, those very things that underlie our selfhood-intimate feelings, childhood memories, amorphic phantasies, dreams, and insights — can be only retrieved from our minds in the linguistic format. Language remains the only operational point of access to the minds of others, all the more so, since the possibility of appropriating someone else's mental states, that is, of the literal "mind reading", has been firmly rejected many times over, both empirically and philosophically².

There is at least another reason to not deny language the power of expression: a practical observation that despite the seeming ineffability of our sensual domain, even those conversations that start with "Words cannot convey ..." do not typically end in silence. Furthermore, admitting that certain things are "beyond words", "indescribable", etc. remains a legitimate communicative strategy, hence Cordelia's complaint quoted above is a meaningful and informative statement, rather than a simple recognition of defeat. The opposition to the idea that language fails when communicating sensory information might be associated with Searle's renowned principle of expressibility - "whatever can be meant can be said"-that has become somewhat of a foundational maxim of positivist thinking (1969: 19). From its extended formulation it follows that: 1) whatever has a meaning has an expression, 2) this expression is verbal in nature, and 3) each meaning can be conveyed directly - all of these implications, collectively, seem to leave no gap whatsoever between the thinkable and the sayable ${ }^{3}$. Thus, we are hung up between these two equally strong intuitions: that of the language's deficiency and that of its communicative omnipotence. The plausible middle ground, I hold, would admit of the following two theses, which I will further explore: 1) some of our mental contents indeed seem ineffable; 2) this ineffability is only relative, as we are generally able to verbalize them, one way or another. The question, therefore, is: how do we say the unsayable?

\section{Ineffability: Weak and Strong}

The problem at hand might be conceived of, simply, as the "lack of words", but in that case we are confronted with the two meanings of "lack" that need to be carefully discerned. The first sense of "lack" is a temporary, non-principled deficiency (such as the lack of money at a given moment), while its second sense is a permanent, fundamental unavailability (such as the lack of the third eye in humans). Hence, depending on which kind of "lack" we have in mind, we will deal with either "weak" or "strong" ineffability of mental contents. Clearly, the latter is of greater interest, however, I will look into the weak version first just to properly set the stage for its more complex variety.

\footnotetext{
${ }^{1}$ If they are not themselves based on language-like codes, like the sign language, etc.

${ }^{2}$ This is not to be confused with another widespread understanding of "mind-reading" in psychology, where it means a form of empathy.

${ }^{3}$ For Searle, however, as well as for the whole of the analytical tradition that he represents, the principle of expressibility is not an empirical truth, but a methodological principle, which does not deny ineffability as such, but treats it as a sign of the speaker's verbal incompetence.
} 
A good way to understand where weak ineffability springs from is to think of a spectrum of color shades. There are, roughly speaking, two ways of representing such spectrum: first, as a neatly organized chart of color samples with clear boundaries between the neighboring shades (each of which can be named), and, second, as an unordered continuum, where shades change one into another at some point without a warning (Fig. 1). The first representation is characteristic of the digital, virtual reality of computer screens, while the second pertains to the analogue world, which we, in fact, inhabit. Here color shades do not come labelled by an authoritative hand, and all distinctions within continuums of degrees remain arbitrary.
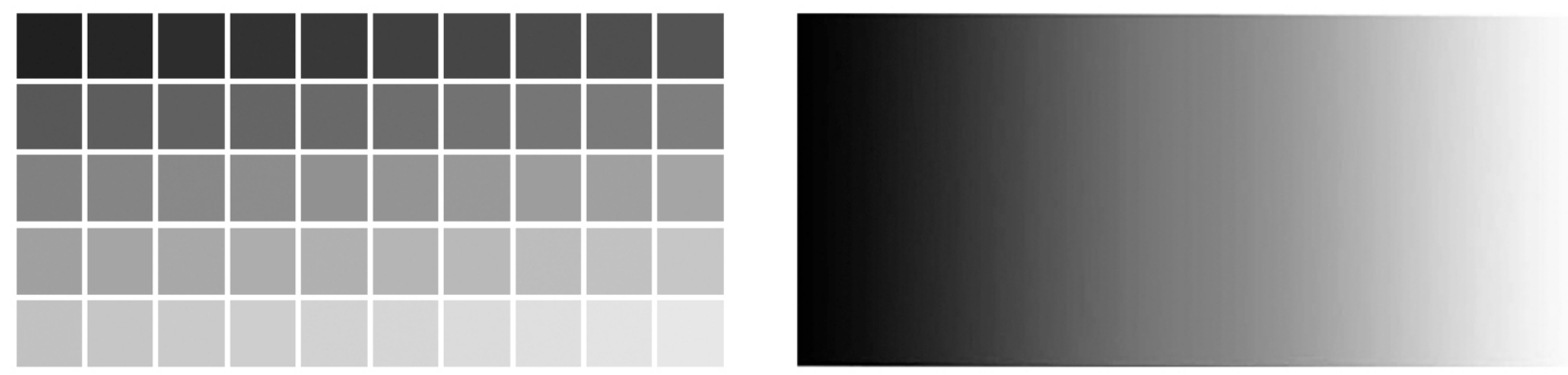

Figure 1. Spectra of gray: "digital" and "analogue" representations

A similar, if not a more pronounced, complication persists when speaking of the sensual domain: while everyday language (and, even more so, the sophisticated medical jargon with its anhedonia, euthymia, cachexia, and a plethora of other terms) does technically discern between, say, anger and rage, sadness and anguish, excitement and happiness, emotional experiences of the sort are still lived as continuums of transient feelings: there cannot be universally accepted criteria for defining at which point exactly anger becomes rage, sadness turns into anguish, and excitement grows into happiness, except for the speaker's inclination to pick up a specific word. This is known as the problem of the ever-increasing "fineness of grain": while digital representations can always be reduced to some kind of "pixel"- the minimal unit, beyond which things are no longer divisible, in real world such fineness of grain is potentially infinite. What it means, in practice, is that though we might invent as many names for color shades, emotions, etc. as we want, we will never be able to have them all named, and all attempts at compiling exhaustive taxonomies for such continuums are inherently misguided.

Thus, weak ineffability means that natural language is fundamentally short of affective predicates, for "there is no set menu of discrete feeling kinds, no periodic table of the heart, ... off-the-shelf categories to specify cleanly and without residue" (Pugmire 1998 qtd. in Pilkington 2001: 4). Hence, we are destined to lack words here and there, and bridging these gaps is a never-ending enterprise. A good demonstration of how weak ineffability is overcome is the "The Dictionary of Obscure Sorrows"- a web-based Borgesian lexicon of mock terms for various emotional experiences with, for instance, the following entries:

lachesism (noun): the desire to be struck by disaster - to survive a plane crash, to lose everything in a fire, to plunge over a waterfall - which would put a kink in the smooth arc of your life, and forge it into something hardened and flexible and sharp, not just a stiff prefabricated beam that barely covers the gap between one end of your life and the other.

vemödalen (noun): the frustration of photographing something amazing when thousands of identical photos already exist - the same sunset, the same waterfall, the same curve of a hip, the same closeup of an eye-which can turn a unique subject into something hollow and pulpy and cheap, like a massproduced piece of furniture you happen to have assembled yourself (The Dictionary ...).

What this and similar projects imply, inter alia, is that given the complexity of the environment that we inhabit, inventing a name for each and every possible mental state that we might hold is a totally senseless enterprise: even if it were theoretically possible, it would simply inflate our everyday vocabulary past the point of its applicability.

Therefore, the problem of inexpressible mental states that I have set forth in the previous section is not about weak ineffability, that is, not about picking (devising, borrowing, etc.) the right names; rather, it is about our seeming inability to describe those states felicitously in words other than generic concepts (love, happiness, sadness, etc.), which do not genuinely refer to the qualitative dimension of our lived experiences. Here comes 
the "strong" ineffability, i.e. the principled lack of verbal structures with which such descriptions could be produced. To better showcase this lack, it will suffice to contrast the way we describe appearances of things with the way we refer to our experiences of interacting with them. In the former case, since entities have associated external properties (or, to put it more accurately, are perceived as having them), we are equipped with a sizeable number of options, such as color, size, shape, smell, taste, etc.: describing entities, thus, is nothing but naming these properties. With experiential states, we evidently lack this convenience, and the use of such attributes will be necessarily figurative-think of black anguish, bitter sadness, or big hopes. Thus, a practical complication arises when attempting to give a direct qualitative description of sensory experiences, which may be illustrated by collating the following pairs of questions, where those on the left are about appearances of entities and those on the right are about experiences associated with them:

$\begin{array}{lll}\text { What does wine taste like? } & - & \text { How does it feel to drink wine? } \\ \text { How does Earth look from space? } & - & \text { What did you feel when you saw Earth from space? } \\ \text { How do I look? } & -\quad \text { What does it feel like to be you? }\end{array}$

Of course, this is still possible to answer the questions on the right in a straightforward, non-figurative manner by using the generic attributes like weird, good, bad, funny etc., which, however, do not deliver conclusive descriptions either in case of appearances or in case of experiences: there is, evidently, a substantive difference in the communicative effect between, say, "I saw a weird dog" and "I saw a blue dog", or between "I am feeling bad" and "I am feeling like I've drunk too much". Trivial statements of the former sort are a staple feature of our everyday, casual communication, which rarely presupposes the need to get circumstantial; but the moment we try to be a little more specific about what exactly weird, good, bad, or funny was about a particular occurrence, we are brought back to our original concern of ineffability. Now, to move forward, I need to place this complication in a suitable epistemological framework involving a few helpful notions from philosophy of mind and cognitive science.

\section{Phenomenal Experiences}

As sentient beings, we undergo all sorts of sensory experiences thanks to our innate abilities of seeing things, hearing sounds, having emotions, etc. Each and every one of these experiences has a subjective qualitative feel: put otherwise, it always feels a certain way to undergo them. When I look at the blue sky on a clear day, or sip cold beer after a long walk in summer, or listen to birds chattering in a grove, I get to know what it feels like to do and experience all those things without much of a cognitive effort: rather, this happens as a natural consequence of just my being conscious. Moreover, I can retrieve this kind of feelings from memory or recreate them through imagination, I can recognize them once they overwhelm me again, and I can surely tell one from another: that is, if my perceptual dispositions are intact, I will maintain that drinking cold beer feels different from drinking warm milk, let alone from driving a car, or hearing growls of thunder.

In the contemporary philosophical parlance, the subjective awareness of the sort is dubbed as phenomenal, while the experiential qualities that it involves are typically referred to as qualia. They have been of little concern to the analytical tradition before the publication of Nagel's seminal essay "What Is It Like to Be a Bat?" in 1974, where he made a point that qualia are quintessentially private and empirically unobservable: to use his example, we cannot know what it feels like to be a bat despite our profound theoretic understanding of its perceptual access to the world through echolocation. Nagel's idea has a controversial metaphysical impact, since he suggests that positivistic science has no means whatsoever to include this phenomenology into its picture and that it has no methodology to subject the phenomenal character of our mental states to any kind of formal analysis (1974: 435-450).

Another famous thought experiment proved helpful in shifting the focus of the qualia debate from metaphysics to epistemology: in the "Knowledge Argument" designed by Jackson in 1982, we are to imagine Mary, a young woman of exceptional scientific rigor and intellect, who studies the neurophysiology of color and, eventually, comes to know everything there is to know on the subject of color perception in human beings. But Mary has a predicament, and a serious one: since her very birth she has been locked up in a black and white room with no hint of colored objects in sight, so, throughout her life, she could only see various shades of grey ${ }^{4}$. When Mary was finally released from her achromatic environment into the outside world, she looked

\footnotetext{
${ }^{4}$ Some argue that the experiment is ill-designed for the purpose: in fact, Mary could have color experiences, for instance, in her dreams, or when rubbing her eyes, etc. By consensus, however, thought experiments do not have to be so rigorously realistic to remain argumentative.
} 
at the sky and could not help exclaiming: "Oh, now I know what it is to see the blue!" (1982: 127-136). The question, therefore, is: did Mary indeed-from just seeing the blue of the sky-learn new facts about the physical world that she could not have known before? If the answer is "yes", then we have to admit, inter alia, that no verbal descriptions of "seeing the blue" could, in principle, help Mary acquire the associated qualia, i.e. that this basic perceptual experience is ineffable in the strong sense.

It has become a widespread convention in the philosophy of mind to suggest that each time we undergo experiences like these, we bring the sensory information that they carry under "phenomenal concepts", which "we exercise when ... we notice or become aware of the phenomenal character of our experiences and feelings through introspection" (Tye 2009: 56). The simplest way to mold or acquire phenomenal concepts is to attend to what is happening here and now-what it feels like to touch a wooden desktop, hear street noises coming from the open window, or suffer from strain in the shoulders-and seize this particular feel in the form of a relatively articulate thought, even if it is nothing more elaborate than "here it goes again". Phenomenal concepts are usually treated as recognitional or indexical thoughts (Loar 1996; Perry 2001), which might be conceived of, schematically, as "I have this feeling of $\mathbf{X}$ ", where $\mathbf{X}$ is the experience that triggers phenomenal awareness. Moreover, most physicalist and dualist ${ }^{5}$ accounts of phenomenal consciousness generally assume that such concepts are pre-linguistic entities, or, more specifically, "the most basic and oldest phylogenetic components of conscious thinking, which preceded the development of language and propositional concepts by many millennia" (Musacchio 2004: 411). In this framework, phenomenal knowledge draws from "analogue neural processes", while our public language is a "digital" instrument that codifies the analogue reality with a varying degree of fidelity: there is always some irreducible information out there that it cannot represent propositionally, and the contents of qualia appear to be of exactly this kind (Jakab 2000). But despite the overall plausibility of these suggestions, we do not reach a deadlock here: for what we know, the said incongruence notwithstanding, we are still able to effectively communicate phenomenal contents, one way or another.

\section{Relativizing the Ineffability}

As I have noted in the section on weak and strong ineffability, nothing prevents us from giving direct qualitative descriptions of our sensory experiences via generic predicates (good, bad, strange, pleasant, etc.), which carry notably less information than the specific attributes with which we refer to external properties of things. Despite their apparent triviality and lack of detail, such utterances are still able of producing a sensible communicative effect as many, if not most, of the experiences that we undergo are of a shared nature, hence, when reporting them casually we except our interlocutors to have the grasp of what it feels like, for instance, to "be caught into a pouring rain", "fly in a shaky plane", or "have a toothache". This is known as human endowment, i.e. the largely shared nature of our phenomenology, which enables us to verbally transfer all types of sensory data in the form of conventionalized approximations.

Things vary, of course, across genres and speech modes, and may run havoc when this endowment is somehow compromised ${ }^{6}$, or when the style and subtlety of such descriptions is of major concern, as is often the case in prose and poetry. As Dennett observes on the subject, the task of mitigating the said conceptual isolation between perception and language by purely verbal means is feasible, for "what it feels like to see Paris by moonlight in May can be adequately conveyed in a few thousand words (an empirical estimate based on the variable success of actual attempts by novelists)" (2005: 115). Said otherwise, the strong ineffability of phenomenal contents is only relative: though direct non-trivial descriptions of our phenomenology may be inconceivable, we can still communicate it through "terms of associated external properties, or ... associated causal roles" (Chalmers 1996: 20-my italics), that is, through the use of analogy: this very insight is, in fact, incorporated into the title of Nagel's essay and the alternative term for qualia: the "what-it's-likeness".

A good example of how this analogical model is utilized is the so-called "TFW" ("this/that-feeling-when") memes, widespread on social media (Miriam-Webster; Know Your Meme):

\footnotetext{
${ }^{5}$ That is, in those accounts that conceive of qualia as entities of their own right, not as just a cognitive illusion.

${ }^{6}$ For instance, when dealing with uncustomary, deviant, or imaginary experiences (flying in space, taking uncommon drugs, experiencing altered states of consciousness, etc.).
} 

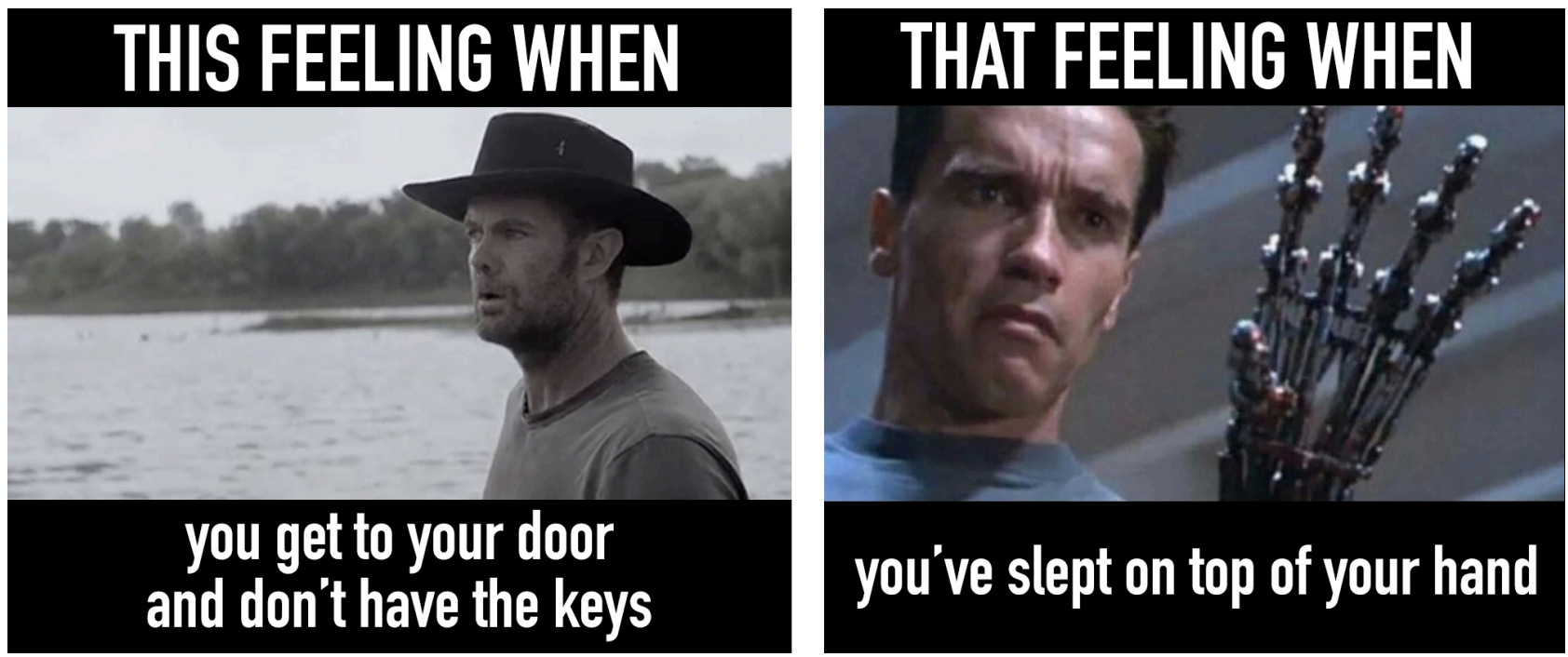

Figure 2. "TFW" memes

In both memes above, the focus is undoubtedly on the qualitative dimension of specific experiences, i.e. on what it feels like to undergo them, while their communicative effect is based on an irony-clad pictorial analogy, drawn, typically, from widely circulated mass cultural artifacts-TV show, music video, film, or commercial stills (an issue that will not interest me here and deserves a separate study). Thus, the meme on the left suggests that reaching the door of your accommodation and realizing that you do not have the keys to open it feels like standing in front (or in the middle) of a large body of water that needs to be crossed; in the meme on the right, the sensation one gets after having slept on her hand is likened to having a robotic limb. All TFW memes share the same basic structure and represent, I posit, the prototypical model of communicating the phenomenal character of sensory experiences through novel metaphors, similes, and metonymies. The rest of the paper will deal with some aspects of how phenomenal character is conveyed in public language through verbal analogy, assuming that the strategy of direct naming is inherently lacking.

\section{Phenomenal Judgments and Phenomemes}

In philosophy of mind, verbal structures used to communicate one's private mental contents are known as "phenomenal judgments", which can be understood, rather loosely, as "words, discussions, and texts about consciousness, subjective phenomena, and the mind-body problem" (Argonov 2014: 53), or more restrictively, as statements containing explicit experiential references—such is the approach of Chalmers, who discerns between the three types (or orders) of phenomenal judgments, exemplified as: "I have a throbbing pain now' ... 'LSD gives me bizarre color sensations' ... 'The problem of consciousness is utterly baffling'” (1996: 161). In Chalmers' paradigm, phenomenal judgments may either assert the fact of undergoing a sensory experience (Type 1), or grasp its qualitative dimension (Type 2), or articulate generalizations on account of consciousness (Type 3). Clearly enough, I will be concerned with only Type 2 phenomenal judgments, since Types 1 and 3 do not elaborate on the phenomenal character of a particular experience as such; moreover, in light of what I have proposed in the previous sections, to be of interest, instead of relying on direct predicates, these judgments need to include analogical assimilations of thematic experiences to something else. I suggest dubbing the discursive unit that emerges through such assimilation as a phenomeme, i.e. a statement that can be parsed to the following prototype:

\section{Experience A is (like) Experience B,}

which is, evidently, a variety of novel metaphor ${ }^{7}$ (or metonymy), where the equivalent of the target element (Experience A) is the caption (C) and the equivalent of the source element (Experience B) is its descriptor (D), e.g. ${ }^{8}$ :

\footnotetext{
${ }^{7}$ The formal difference between similes and metaphors might be ignored for the purposes of this study, as they are structurally reducible to each other.

${ }^{8}$ The source for all examples below, if not specified otherwise, is "Handmaid's Tale" by M. Atwood (1986).
} 
(1) Still, I was angry, as if I'd been unjustly accused of something I didn't even know about (152).

C (being angry):

D (being wrongfully accused)

(2) Quiet, I say again, my face is wet, sweat or tears, I feel calm and floating, as if I'm no longer in my body (64).

C (calmness):

D1 (floating)

D2 (being outside one's body)

A single phenomenal judgment-which is not always equivalent to a sentence, but may span over an arbitrary length of text-may contain several distinct phenomemes, in which, typically, two or more descriptors "cling" to the caption, thus creating multiple alternative reiterations for the same target element, e.g.:

(3) The sexual act, although he performed it in a perfunctory way, must have been largely unconscious for him, like scratching himself. It was like being on an operating table, in the full glare; like being on a stage (138).

C (having sex):

D1 (scratching oneself)

D2 (being on an operating table)

D3 (being on stage)

In another recurrent model of phenomemes with several descriptors, the latter stand in the subordinate relations with each other, thus creating a phenomeme within a phenomeme, with the "master" descriptor functioning as an auxiliary caption, e.g.:

(4) Time has not stood still. It has washed over me, washed me away, as if I'm nothing more than a woman of sand, left by a careless child too near the water ... A shadow of a shadow, as dead mothers become (73).

C (being forgotten by one's child):

D1 (being washed away by time):

d1 (being a sand figure destroyed by water)

D2 (being a shadow of a shadow):

d2 (being a dead parent)

As a matter of fact, such explicit phenomemes might be evaluated along a number of dimensionsprimarily, those already at play in the general theories of metaphor, simile, and metonymy-but I will first categorize them along the following axes: degree of complexity, which refers to whether or not their captions rely on public concepts, and degree of stability, i.e. their reducibility to existing conceptual metaphoric or metonymic models. I will elaborate on this tentative taxonomy in the two sub-sections below.

\subsection{Captions: Degree of Complexity}

At first view, phenomemes seem to differ in the general structure of their captions, which could be either simple, i.e. rely on the generic concepts of the public language--for instance, names of emotions, perceptual, bodily, or interoceptive sensations ("love", "sadness", "happiness", "pain", "hunger", etc.)—or complex, i.e. not easily reducible to such labels, e.g.:

(5) We looked at one another's faces and saw dismay, and a certain shame, as if we'd been caught doing something we shouldn't (153).
C (shame):
D (being caught in the middle of wrongdoing)

(6) I want to reach up, taste his skin, he makes me hungry (85).

C (lust):

D (desire to touch):

d (hunger) 
Both (5) and (6) above contain simple phenomemes, as their captions are public concepts of "shame" and "lust" (or "sexual arousal)" accordingly. In contrast, the complex captions of (7) and (8) below can be only conceived of as situations or specific occurrences:

(7) I follow the downstairs corridor back, past the door that leads into the kitchen, along to the next door, his. I stand outside it, feeling like a child who's been summoned, at school, to the principal's office (118).

C (standing in front of a lover's door):

D: (standing in front of the principal's office as a school child)

(8) I sink down into my body as into a swamp, fenland, where only I know the footing. Treacherous ground, my own territory. I become the earth I set my ear against, for rumors of the future. [...] Each month I watch for blood, fearfully, for when it comes it means failure (63).
C (checking the signs of pregnancy):
D1: (sinking down into one's own body)
D2: (listening to one's body from within)

Categorization of phenomemes as either simple or complex might involve a degree of arbitrariness and rely on the study of the immediate and/or larger context (as in (5)). This is due to the fact that captions may appear elliptical, that is, implied rather that overtly verbalized. In this case, if the candidate caption safely resolves itself to a public concept, like in (9), where the overall context and the closing remark "I want to be with someone" allude expressly to some form of "loneliness", it is considered simple; if it resists such reduction, like in (10), it is classified as complex:

(9) You can wet the rim of a glass and run your finger around the rim and it will make a sound. This is what I feel like: this sound of glass. I feel like the word shatter. I want to be with someone (88). C (loneliness):

D (being a sound produced by running a finger along the rim of a glass)

(10) I have been obliterated for her. I am only a shadow now, far back behind the glib shiny surface of this photograph. [...] I can't bear it, to have been erased like that (197).

C (being forgotten by one's child): $\quad$ D1 (being a shadow)

D2 (being erased)

\subsection{Descriptors: Degree of Stability}

Descriptors reiterate captions to form novel metaphors and metonymies, and, in this capacity, they may perform either of the two principal discursive functions: explicative, i.e. rendering abstract, complex, or deviant captions via more concrete and relatable representations; or, in reverse, complicative, i.e. elevating trivial captions to a more sophisticated and original imagery. Admittedly, the only guideline in establishing such function is one's own intuition, since the discursive markers that could help disambiguate the choice are not readily available. However, what can be ascertained reliably enough in this connection is whether descriptors can be reduced to well-established conceptual models that are rooted in language in the form of idiomatic or set expressions, or whether they somehow disrupt the extant patterns and set new associations in motion. In the former case, it makes sense to speak of stable phenomemes, e.g.:

(11) The moment of betrayal is the worst, the moment when you know beyond any doubt that you've been betrayed [...] It was like being in an elevator cut loose at the top. Falling, falling, and not knowing when you will hit (67).
C (feeling of betrayal):
D (being inside a falling elevator)

(12) Some days I do appreciate things more, eggs, flowers, but then I decide I'm only having an attack of sentimentality, my brain going pastel Technicolor, like the beautiful-sunset greeting cards they used to make so many of in California (171).
C (sadness):
D (seeing things in Technicolor) 
In (11), the generic episode of "betrayal" is portrayed as "being inside a falling elevator", which may be generalized as "losing one's ground"-a model that figures prominently in many English idiomatic expressions, to wit: "to cut the ground from under someone's feet", "to knock the bottom out of someone", or "to pull the carpet from under someone". In a similar vein, the descriptor of (12), where "sadness" (or "depression") is likened to "seeing things in Technicolor", can be recast as "loss of color (vision)", which is salient in such expressions as "to be in gray moods", or, on the contrary, "to see things in full color", etc.

The descriptors of (13) and (14) below do not follow the existing models and propose original (if not counterintuitive, as in (3)), off-the-wall imagery, which might require a larger context in order to be grasped. These phenomemes are, thus, of the unstable type:

(13) It makes me feel slightly ill, as if I'm in a closed car on a hot muggy day with an older woman wearing too much face powder (68).
C (being ill):
D (being in a closed car on a hot day with an older woman who wears too much face powder)

(14) Winter is not so dangerous. I need hardness, cold, rigidity; not this heaviness, as if I'm a melon on a stem, this liquid ripeness (131).

C (feeling hot):

D (being heavy):

d (being a melon on a stem)

Whether simplicity/complexity correlates somehow with stability/instability can be confirmed or disproved by studying a larger corpus and by taking some additional factors (for instance, the semantic class of captions) into account, which is a prospect for further work.

\section{Phenomemes in Discourse}

The study of qualia within linguistic settings might sound like a novel enterprise, however, there is a relatively long and vibrant history of research in the adjacent fields-for instance, of sensory vernaculars and the associated communicative strategies - as testified by the works of Bucci, Langer, Paivio, and Scherer, to name but a few. The diversity and impact of these studies notwithstanding, they tend to share one common underlying principle, if not prejudice: the overvaluation of verbal constraints on the communication of qualitative states. A typical project within this paradigm would build on the strong ineffability of sensory inputs and, sometimes, even call for construing "a language at the interface of science and poetry that may serve as a better approximation to qualia than is offered by declarative or propositional statements and yet amenable to formalization and quantification" (Zentner 2012: 81) ${ }^{9}$.

Evidently, such studies are headed into a radically different direction from my proposal, that is, from positing that the relative ineffability of phenomenal contents does not play down the ability of public language to communicate qualia. In fact, it seems that the need to somehow cope with this ineffability made language an extremely flexible and creative tool and led to a significant upgrade in the capacities of human mind ever since linguistic competence had become a part of its repertoire. My idea here is to highlight that language is fully capable of conveying phenomenal contents of all sorts contrary to the prevalent assumption that it only "names certain vaguely and crudely conceived states, but fails miserably in any attempt to convey the evermoving patterns, the ambivalence and intricacies of inner experience, the interplay of feelings with thoughts and impressions, memories and echoes of memories, transient fantasy, or its mere runic traces..." (Langer 1942: 100). To refute this verdict of the "miserable failure" of language in communicating qualia or, at the very least, to make this allegation susceptible to further argument, it will suffice to have a quick look at the literary discourse, in which phenomenal states - however subtle and elusive - are conveyed through a web of analogies, for instance:

(15) I sink down into my body as into a swamp, fenland, where only I know the footing. Treacherous ground, my own territory. I become the earth I set my ear against, for rumors of the future. Each twinge, each murmur of slight pain, ripples of sloughed-off matter, swellings and diminishings of tissue, the droolings of the flesh, these are signs, these are the things I need to know about. Each month I watch for blood, fearfully, for when it comes it means failure (63).

\footnotetext{
${ }^{9}$ The cited paper explores the possibility of creating a specialized vernacular more suited to communicate musicassociated qualia.
} 
One legitimate objection that may arise in this connection is that though ineffability of qualia seems manageable in written texts, it might persist in the spoken register: as Bucci et al. reveal in their study, when asked to verbally elaborate on their emotional states, most speakers first undergo the stage of arousal, that is, of resorting to gestures and facial mimics combined with or followed shortly by reporting the inability to fulfill the task immediately due to the lack of words (2016: 373). Perhaps, no clinical trials are needed to infer that grasping the right sort of vocabulary and reconceiving imaginatively of one's own feelings and sensations, let alone of qualitative states, is indeed a challenge that most speakers are unable to tackle on the spur of the moment. Moreover, I maintain that the problem of verbalizing perceptual contents is simply peripheral for colloquial speech, where reliance on human endowment takes precedence over the conceptual subtleties in questions. As a consequence, all sensible analysis of phenomemes should proceed from written genres, where the stage of arousal and the associated verbal confusion are typically not part of the message.

The role of phenomemes thus understood goes far beyond framing immediate contexts: rather, they constitute a textual dimension of their own and evolve into a narrative technology of portraying "subjective selves" that immerse the audience into a web of fictional phenomenologies. As Camp notes in her speculations on "Anna Karenina", L. Tolstoy's ambition throughout the many pages of his symphonic novel was to cause readers to identify with the main protagonist, which means "transforming [themselves] imaginatively, so that [they] become Anna Karenina... which involves imagining [themselves] being Anna Karenina, by imaginatively altering the content of who [they are]: endowing [themselves] with properties that Anna possesses which [they] lack..." (2009: 112). I hold that such identification is achieved, primarily, through referencing the protagonist's qualia, that is, by using phenomemes instead of the existing nomenclature of emotional and sensory states, which only invokes a third-person perspective-an observer's stance, which is better suited for scientific descriptions and other non-fictional discourses. Taming the ineffability of what-it'slikeness can thus be seen as a path to a more intimate involvement with what is normally inaccessible: the mind of the Other. It has been widely accepted in the post-Gricean pragmatics of communication that such "mind-reading", i.e. grasping and felicitously interpreting the speaker's meaning, lies at the core of mutual understanding as such (Watzlawick et al.2014). But it is only in literature-a laboratory of devising new and reiterating the existing tools of communicating the unsayable-where this communicative experience is of unmatched aesthetic and redemptive value. In this view, the problematics of phenomenal discourse should figure prominently across the field of literary and pragmatic studies, since, as Pilkington notes:

'Poetic concepts' and 'poetic thoughts' ... are difficult and relatively ineffable for a special reason: they encourage extensive encyclopaedic exploration and elaborate concept construction that involves the evocation of intense and subtle qualitative states. Such qualitative states cannot be described satisfactorily: they are non-lexicalisable. But insofar as they are effable, they evoke/arouse rather than simply characterise/describe an experience. Any attempt at a full discussion of poetic effects and poetic thoughts must discuss the evocation of qualitative states, and to this extent must reach out beyond pragmatic theorising (2001: 9).

\section{Conclusion}

The problem of ineffability of phenomenal contents is firmly rooted in the interdisciplinary problematics of the "explanatory gap" between physicalism and phenomenal consciousness. While philosophers will, perhaps, never stop debating about the ontological consequences of the said gap, its epistemological effects can be assessed through the lens of the human language capacity and pragmatics of communication. Making sense of this sophisticated philosophical landscape seems a major challenge to tackle, but I hold that acknowledging its discursive and narrative dimensions within the framework of cognitive poetics, which "[incorporates] the insights of cognitive science into literary studies" (Hart 2001: 314), can lead to sensible insights on the matter. It is, primarily, fictional prose and poetry - not spoken speech that is often preferred in cognitive studies for being a more "organic" medium of verbal thinking — which offers a wealth of evidence to weigh and to develop an understanding of how the qualitative dimensions of sensory experiences, or "qualia", are conveyed effectively in public language despite their privacy and seeming inexpressibility.

Within the confines of this paper, the ineffability of phenomenal contents is relativized by invoking the notion of phenomenal judgments, or "phenomemes", that exhibit a number of structural and semantic regularities. As I briefly note in the closing section, phenomenal discourse constitutes a narrative technique, which propels the affective engagement of readers with texts and brings the paradigm of text interpretation as a mind-reading activity into focus. It is my hope that these preliminary findings might grow into a more 
substantial research and be a step forward in establishing the scope to which language affects or predetermines our cognitive performance.

\section{References}

Argonov, V. 2014. "Experimental Methods for Unraveling the Mind-Body Problem: The Phenomenal Judgment Approach." The Journal of Mind and Behavior 35 (1/2). 51-70.

Atwood, M. 1986. Handmaid's Tale. New York: O. W. Toad Ltd.

Berta, L. 2011. "Qualia from the Point of View of Language." The Journal of Mind and Behavior 32 (5). 181-208.

Bucci, W. et al. 2016. "Connecting Emotions and Words: The Referential Process." Phenomenology and the Cognitive Sciences 15.359-383.

Camp, E. 2009. "Two Varieties of Literary Imagination: Metaphor, Fiction, and Thought Experiment." Midwest Studies in Philosophy 23. 107-130.

Carruthers, P. 1996. Language, Thought, and Consciousness. Cambridge: Cambridge University Press. Chalmers, D. 1996. The Conscious Mind: In Search of a Fundamental Theory. Oxford: Oxford University Press.

Dennett, D. 2005. Sweet Dreams. Philosophical Obstacles to the Science of Consciousness. Cambridge, MA: The MIT Press.

Hart, E. F. 2001. “The Epistemology of Cognitive Literary Studies.” Philosophy and Literature 25 (2). 314 334.

Jackson, F. 1982. “Epiphenomenal Qualia.” Philosophical Quarterly 32. 127-136.

Jakab, Z. 2000. "Ineffability of Qualia: A Straightforward Naturalistic Explanation." Consciousness and Cognition 9 (3). 329-351.

Know Your Meme [Accessed 09 April 2020]. https://knowyourmeme.com/memes/i-know-that-feel-bro Langer, S. K. 1942. Philosophy in a New Key. New York: Penguin.

Loar, B. "Phenomenal States.” In: Tomberlin, J. (ed.). 1996. Philosophical Perspectives 4: Action Theory and Philosophy of Mind. Atascadero: Ridgeview. 81-108.

Miriam-Webster Online Dictionary and Thesaurus. [Accessed 09 April 2020]. https://www.merriamwebster.com/words-at-play/what-does-tfw-mean-that-feeling-when

Musacchio, J. M. 2004. "The Ineffability of Qualia and the Word-Anchoring Problem.” Language Sciences 27. 403-435.

Nagel, T. 1974. "What Is It Like to Be a Bat?" Philosophical Review 8 (4). 435-450.

Nordmann, A. 2005. Wittgenstein's Tractatus: An Introduction. Cambridge: Cambridge University Press.

Perry, J. 2001. Knowledge, Possibility, and Consciousness. Cambridge, MA: The MIT Press.

Pilkington, A. 2001. "Non-Lexicalised Concepts and Degrees of Effability: Poetic Thoughts and the Attraction of What Is Not in the Dictionary." Belgian Journal of Linguistics 15 (1). 1-10.

Searle, J. R. 1969. Speech Acts. Cambridge: Cambridge University Press.

Shakespeare, W. 2009. King Lear. Cambridge: Cambridge University Press.

The Dictionary of Obscure Sorrows. [Accessed 14 November 2020].

https://www.dictionaryofobscuresorrows.com

Tye, M. 2009. Consciousness Revisited. Cambridge, MA: The MIT Press.

Watzlawick, P. et al. 2014. The Pragmatics of Communication: A Study of Interactional Patterns, Pathologies, and Paradoxes. New York: WW Norton and Co.

Wittgenstein, L. 2009. Major Works. Selected Philosophical Writings. New York: HarperCollins Publishers. Zentner, M. 2012. “A Language for Musical Qualia.” Empirical Musicology Review 7 (1/2). 80-83.

Zlatev, J. 2008. "The Dialectics of Consciousness and Language." Journal of Consciousness Studies 15 (6). $5-14$. 